\title{
INCORPORATING INFORMATION NETWORKS INTO MILITARY SIMULATIONS
}

\author{
Darryl K. Ahner \\ Department of Mathematical Sciences \\ U.S. Military Academy \\ West Point, NY, 10996, U.S.A. \\ John Jackson \\ Joint Concept Development \& \\ Experimentation Division \\ U.S. Joint Forces Command J9 \\ Suffolk, VA, 23435, U.S.A.
}

\author{
Jonathon K. Alt \\ Francisco Baez \\ U.S. Army TRADOC Analysis Center \\ P.O. Box 8695 \\ Monterey, CA, 93943, U.S.A. \\ Susan M. Sanchez \\ Thorsten Seitz \\ Operations Research Department \\ Naval Postgraduate School \\ Monterey, CA, 93943, U.S.A.
}

\begin{abstract}
Information superiority is considered a critical capability for future joint forces. As advances in technology continue to boost our ability to communicate in new and different ways, military forces are restructuring to incorporate these technologies. Yet we are still limited in our ability to measure the contributions made by information networks. We describe three recent studies at the Naval Postgraduate School that involve information networks. First, we examine a simulation model expanded from a two-person, zero-sum game to explore how information superiority contributes to battlefield results and how sensitive it is to information quality. Second, we examine how network-enabled communications affect the logistics operations in a centralized receiving and shipping point. The results are intended to provide operational insights for terminal node operations within a sustainment base. Third, we explore how social networks might be incorporated into agent-based models representing civilian populations in stability operations.
\end{abstract}

\section{INTRODUCTION}

For many decades, Department of Defense (DoD) and other national defense analysts have studied potential large-scale warfare between states. The results of these analyses often inform decisions on how nations should build, organize, maintain, and, if necessary, employ their military and security forces. Since, happily, there is a dearth of such conflicts, most of this analysis has been and is being done via experimentation. Because of the expense and other constraints associated with live experimentation, much of this experimentation is done with simulation. With the increasing reliance on new technologies and network-enabled operations, incorporating information networks into the simulation models used to support these decisions is important.

In this paper, we describe three recent studies at the Naval Postgraduate School that involve information networks. The three examples are quite different in how they model aspects of information networks, as well is in the application areas. Yet one commonality to all these studies is that they do not build a single, baseline model and stop. Instead, they use efficient experimental designs to conduct a broad exploration of the simulation models' behaviors under various conditions.

\section{ASSESSING THE VALUE OF INFORMATION SUPERIORITY}

Proponents view information superiority as a force multiplier; given forces of equal size and ability, the one that possesses information superiority can achieve superior results to those of the other. Research suggests that this is, in fact, the case. Yet, what are the risks associated with units relying on information superiority? How can we measure the degree of superiority that an information advantage provides? How much is enough? In a world constrained by budgets, these questions must be addressed so a proper balance can be made between equipment meant to destroy our adversaries and equipment that facilitates information superiority. It has been aptly pointed out by General Howell M. Estes III, USAF, a former commander of Space Com- 
mand that, "you can't take out an enemy tank with just information" (Washburn 2001).

Unfortunately, attacking this type of problem has proven difficult for the operations research community. As Washburn (2001) says, "There is a crisis for military OR, centered on the role of information on the battlefield. It is clear to military professionals that information is becoming increasingly important, but unfortunately the OR profession's ability to measure its contribution is still primitive."

We do not attempt to make a breakthrough in these "primitive" measures, but, instead, creatively apply several methods to address the following questions:

- How do varying degrees of information superiority affect battlefield outcomes?

- How sensitive are these outcomes to the quality of information used to obtain information superiority?

- Given a certain level of success achieved through information superiority, what increase in force size is required to achieve similar results if information superiority is taken away?

The link between information superiority and a decision maker is key. Information superiority is the "advantage gained by the ability to collect, process, and disseminate an uninterrupted flow of information while exploiting or denying an adversary's ability to do the same" (Joint Chiefs of Staff 2006), but this advantage is turned into improved battlefield success through the decision-making process.

We implement a decision model based on a two-person zero sum (TPZS) game (Bracken and Darilek 1998; Washburn 2003). These games are an excellent abstraction of military conflict since they involve two opposing sides, each of whom must choose from an array of strategies in order to achieve the most desirable result.

\subsection{Structure of the Game}

We describe the decision model with the help of Figure 1, which we refer to as the game board. There are two sides (red and blue), and three objectives (rectangles between columns four and five) that represent locations where red and blue will encounter one another.

On the game board, both red and blue begin with all their forces at their respective start locations. Red will advance one column per move until it reaches column 4. Blue will deploy its forces over time into column five. Blue has the opportunity to use a sensor placement strategy to collect information about red movement. Red wins an encounter in a given row when their forces in column four of that row outnumber the blue forces in column five of the same row.

For a particular instance of game play, the value $v_{i}$ associated with the objective in row $i$ depends on the number of red and blue forces present in rows four and five,

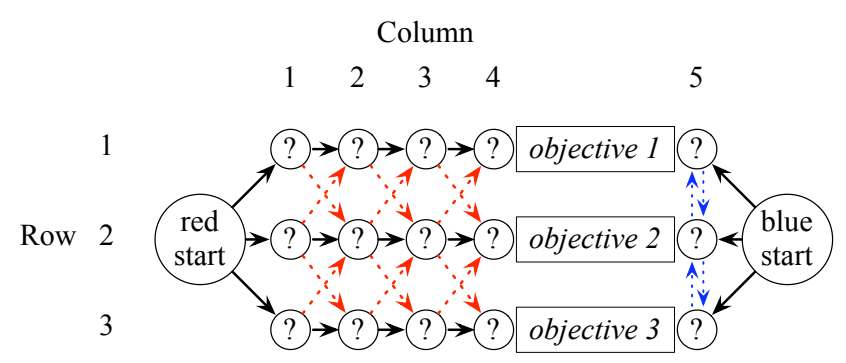

Figure 1: Graphic depiction of the game board (adapted from Jackson 2008).

respectively, when the engagement occurs. Let $R_{i}$ and $B_{i}$ denote these values for objective $i$. The total red score is $\sum_{i=1}^{3}\left[R_{i}-B_{i}\right]^{+}$, where $y^{+}=\max (y, 0)$. Red tries to maximize this score by attacking the objectives, while blue tries to minimize it by defending the objectives.

\subsubsection{General Conditions}

Both red and blue understand the rules of the game. Red knows the number of blues and the restrictions of movement that pertain to blue, but will not be given any other information. Specifically, red will not find out whether are present during the course of play.

Blue understands the restrictions on red movement, but does not necessarily have accurate knowledge about the total number of red units. Blue does not know what strategy red will employ, but will try to determine this based on information obtained from sensors. This allows blue to adjust its strategy as time progresses, so blue will want to remain as flexible as possible within the rules of the game.

\subsubsection{Red Strategy}

In a TPZS game with information parity (i.e., where neither side has information as to how the other will behave), the optimal action for each side is to play their respective strategies with the proportion that guarantees the maximum expected value, regardless of the strategy the adversary selects. This is known as the optimal mixed strategy, and serves as the baseline. In this game, the optimal mixed strategy for red can be found by solving a linear programming problem. The optimal mixed strategy involves moving all of red's forces to a single row in column four, yielding three optimal courses of action.

\subsubsection{Red Movement Restrictions}

During its first turn, red must move all of its units from its start point to any positions desired in column one. During subsequent turns, it must adhere to three important rules 
governing its movement: 1) during each turn all red units must move out of one column and into another, highernumbered column; 2) red can move at most one unit across dotted (red) paths; and 3) red can move up to the maximum number of reds present in the previous location in each of the respective rows across solid (black) paths.

\subsubsection{Blue Movement Restrictions}

Blue can move as many units into column five as desired in blue's initial deployment, but no units can return to the start location once deployed. For each subsequent turn, blue can move at most three units out of its start location; blue can also move at most one unit across each dotted (blue) path between adjacent rows in column five. In practice, blue will hold back some units in order to maintain the maximum level of flexibility, in the hopes that its sensors will provide useful information about where red units will attack.

\subsubsection{Basic Game Play Sequence}

The game progresses after the number of red, number of blue, and number of sensors have been assigned.

1. Red randomly selects an optimal course of action, without knowing blue's disposition. Red's movements can then be predetermined since red will not make any decisions based on the progression of the game. There is an additional stochastic component to the actual movement patterns, since there are many different ways that the red units can disperse but meet up at the final objective.

2. Blue uses a sensor decision algorithm (described in Section 2.1.6) to place sensors on the game board. Only one sensor can be placed per location in columns one through three on the game board.

3. Blue uses a movement decision algorithm (described in Section 2.1.7) to determine the initial deployment of blue units into column five.

4. Red moves into column one according to its predetermined plan, and blue makes its initial movements into column five according to step (3).

5. Blue information is updated (if appropriate); this information may lead to blue adjusting its units in column five subject to its movement restrictions.

6. Play continues-with red using its predetermined movements, and blue using its movement decision algorithm - until the red units are in column four. The red score is calculated and the game ends.

\subsubsection{Blue Sensor Decision Algorithm}

Ideally, blue will use its sensors to maximize its informational advantage. However, determining an optimal sensor array when there are more nodes than sensors proved an intractable task, even when the information was perfect (i.e., no delay and no erroneous information). We attempted to determine if, for a given number of red, blue, and blue sensors, an optimal sensor array could be found using simulation, but did not converge to an optimal sensor array even after conducting a very large number of replications. Therefore, we chose to set up a smaller simulation within the overarching model to select a sensor array for blue that seemed reasonable, even though it may not be optimal. This smaller simulation works similarly to the larger simulation. For a given number of red, blue, and blue sensors, a specific game is played varying the probability of time delay and the accuracy stochastically, while conducting 50 replications of every possible sensor configuration available to blue.

\subsubsection{Blue Movement Decision Algorithm}

The procedure for determining how blue will move into column five is the most complex process, and involves numerous comparisons and updates. The idea is to not commit forces before it is necessary. Blue always elects to keep the maximum number of units at the start location as long as possible, provided none are left there at the end of each game. For future deployments of blue units, blue will look to update its information concerning red's intentions. To do this, blue keeps track of two pieces of information about red: 1) the maximum number of reds possible at each objective, and 2) the minimum number of reds required at each objective. These two values are based on red's movement restrictions. Blue evaluates the differences between the maximum number possible at a particular objective, and the current number of blues deployed to that objective, and seeks to balance the differences evenly across the objectives.

Of course, all of these calculations are influenced by the accuracy and timeliness of information transmission. If there is a delay in sending information, blue loses the opportunity to take advantage of that information. However, prior to the next turn, information about the maximum number of reds possible at each objective is first updated based on the delayed information, then it is updated based on the availability of any new information, and finally blue makes its next deployment of units. There is no direct resolution for any potential conflicting or ambiguous information.

\subsection{Experiment}

The model was implemented in Java with the addition of LPSolve, which is a freeware linear program/mixed-integer program (LP/MIP) solver that is compatible with Java.

The model has five parameters: the number of red $(R)$, number of blue $(B)$, number of blue sensors $\left(S_{B}\right)$, accuracy variation $(A V)$, and probability of time delay $\left(P_{D}\right)$. We evaluate situations where $R=\{2, \ldots, 25\}$ and 
$B=\{1, \ldots,\lfloor 3 R-1\rfloor\}$. (Note that if $B \geq 3 R$, blue can defend each location without any concern that red will score any points. Thus, it is not important to explore this region.)

The number of blue sensors $S_{B}$ varies from 1 to 9 . Only one sensor can be placed at any of the nine discrete locations on the game board, located in columns one through three. Blue is then able to use this information to varying degrees to adjust its course of action.

The quality of the information may be affected by the accuracy variation, which has six distinct levels. The percentages of time that various miscounts of red units occur area are shown (to the nearest percent) in Table 1.

Table 1: Probabilities of miscounting of the number of red.

\begin{tabular}{c|rrrrrr}
\multirow{2}{*}{\begin{tabular}{c|c}
$\mid 7$ \\
Level
\end{tabular}} & 0 & \pm 1 & \pm 2 & \pm 3 & \pm 4 & \begin{tabular}{r} 
Discrepancy in red count \\
\cline { 2 - 7 }
\end{tabular} \\
\hline 1 & $68 \%$ & $27 \%$ & $40 \%$ & $<1 \%$ & $<1 \%$ & $<1 \%$ \\
2 & $38 \%$ & $30 \%$ & $18 \%$ & $9 \%$ & $3 \%$ & $2 \%$ \\
3 & $26 \%$ & $23 \%$ & $19 \%$ & $13 \%$ & $9 \%$ & $10 \%$ \\
4 & $20 \%$ & $18 \%$ & $16 \%$ & $14 \%$ & $11 \%$ & $21 \%$ \\
5 & $16 \%$ & $15 \%$ & $14 \%$ & $12 \%$ & $11 \%$ & $32 \%$ \\
6 & $13 \%$ & $13 \%$ & $12 \%$ & $12 \%$ & $10 \%$ & $40 \%$ \\
\hline
\end{tabular}

The final factor in the model is the probability of a time delay. This ranges from 0.0 to 0.8 . A value of 0.5 means that there is a $50 \%$ chance that a delay will occur. The length of the delay is always only a single turn. Thus, a report that blue expected to receive during its current turn will not be available until the following turn.

Both the probability of time delay and the accuracy variation apply uniformly to each sensor being employed within a specific game (i.e., each sensor will have the same probability of delay and the same accuracy variation). This was done primarily for the sake of simplicity, but the restriction could be relaxed in future studies.

For each specific set of inputs, the game will run for a predetermined number of replications, which will constitute one run of the model. For each run, the model will output a low score, high score, and the average score for red.

A total of 948 combinations of red and blue are examined; for each of these, 99 combinations of the number of sensors, the accuracy variation, and probability of delay are examined, and 50 replications of each of these 93,852 games were conducted.

\subsection{Results}

Jackson (2008) does a detailed analysis of the results using a mix of techniques (metamodeling, graphical analysis, etc.). We provide a few snapshots from his work to summarize some of the key findings. The baseline of comparison is the game value (i.e., expected red score) when no sensors are available and each player uses an optimal mixed strategy; this baseline game value can be determined mathematically.

First, consider Figure 2. For each combination of red and blue units, two average values are computed from the simulation experiment: (i) the game values when no sensors are available, and (ii) the average red scores when blue has one or more sensors, averaged across all the variations of $S_{B}, A V$, and $P_{D}$. For all instances where the number of red units is greater than five, the average red score is lower when blue has sensors. Observe that the magnitude of the improvement is greatest when $B \approx 1.5 R$, and sensors provide the least value when $B$ is near either endpoint of its range ( 1 or $3 R-1$ for a particular $R$ ).

Next, for an overview of the impact of using different numbers of sensors as a function of the number of red and blue units, see Figure 3. The vertical axis displays the scaled decrease in the red score, defined as

$$
D^{*}=[(\text { Game Value })-(\text { Mean Red Score })] / R \text {. }
$$

The patterns in Figures 2 and 3 continue as $R$ rises. In Table 2 we provide slices of the results when $R=17$ for various $B$ to show these ideas more clearly. Results for $S_{B}=6,7$, and 8 (not shown) are similar to those for $S_{B}=5$ and $S_{B}=9$.

Table 2: The value of sensor information for various numbers of blue units $(B)$ and numbers of blue sensors $\left(S_{B}\right)$, when the number of red $(R)$ is 17 .

\begin{tabular}{r|c|rrrrr} 
& Info. & \multicolumn{5}{|c}{ Number of Blue } \\
$S_{B}$ & Value & 6 & 10 & 17 & \multicolumn{1}{c}{27} & \multicolumn{1}{c}{40} \\
\hline \hline 1 & $D^{*}$ & 0.098 & 0.133 & 0.140 & 0.192 & 0.157 \\
& $E B$ & 5.0 & 6.8 & 7.2 & 9.8 & 8.0 \\
\hline 2 & $D^{*}$ & 0.185 & 0.232 & 0.258 & 0.318 & 0.190 \\
& $E B$ & 9.5 & 11.8 & 13.1 & 16.2 & 9.7 \\
\hline 3 & $D^{*}$ & 0.190 & 0.262 & 0.266 & 0.305 & 0.189 \\
& $E B$ & 9.7 & 13.3 & 13.6 & 15.6 & 9.6 \\
\hline 4 & $D^{*}$ & 0.200 & 0.275 & 0.295 & 0.326 & 0.195 \\
& $E B$ & 10.2 & 14.0 & 15.0 & 16.6 & 10.0 \\
\hline 5 & $D^{*}$ & 0.220 & 0.289 & 0.322 & 0.367 & 0.205 \\
& $E B$ & 11.2 & 14.8 & 16.4 & 18.7 & 10.4 \\
\hline 9 & $D^{*}$ & 0.217 & 0.287 & 0.326 & 0.367 & 0.205 \\
& $E B$ & 11.1 & 14.6 & 16.6 & 18.7 & 10.5 \\
\hline \hline
\end{tabular}

Regression analysis allows a more detailed investigation of the how the five factors and their interactions affect the red score. It reveals that nonlinear relationships exist between the value of information superiority and both force ratio and force size. There is an initial minimal force requirement before information has much value. Additionally, once force size reaches a certain point, the value of information superiority begins to decline sharply. 


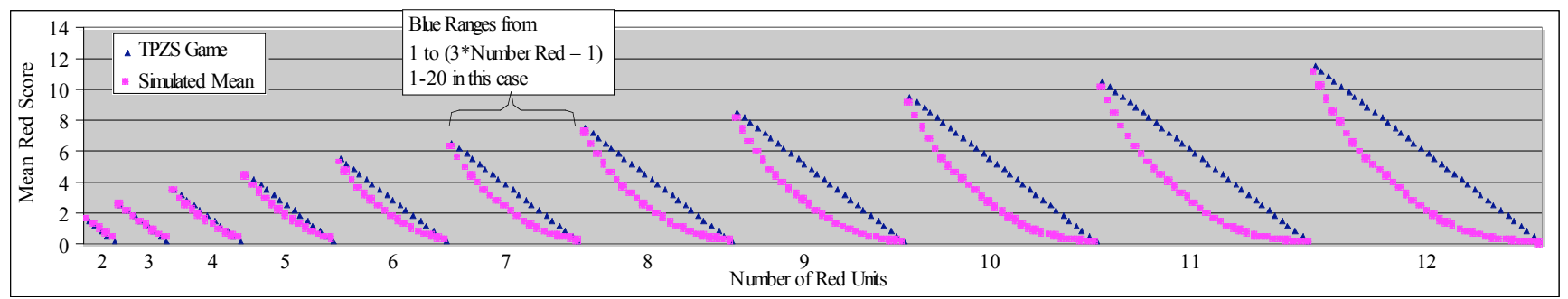

Figure 2: Comparison of average red scores with sensors to expected red scores without sensors.

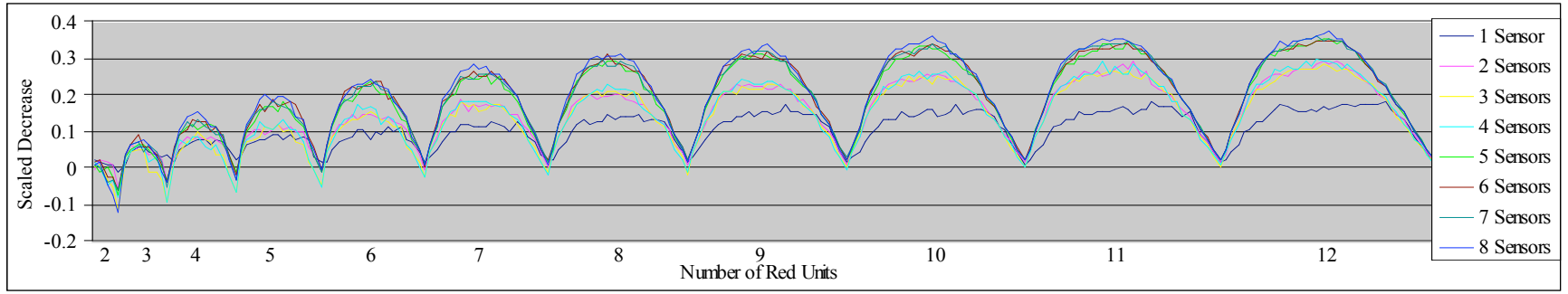

Figure 3: The value of sensor information in terms of the scaled percent decrease in red score.

The experimental results also suggest that decreasing information quality degrades the value of information superiority more uniformly. In this study, poor information quality is only slightly (at best) mitigated by an increased number of sensors. Finally, the results show that while there are risks to relying on information superiority in terms of potential decreases in battlefield performance, there is a much greater potential for increased battlefield performance.

This study explores the contribution of information in a practical manner which yields useful insights. Preliminary results are intriguing, and we feel that this approach has significant potential for further work.

\section{SOCIAL NETWORKS IN A SIMULATION OF URBAN CULTURAL GEOGRAPHY}

Civilian human behavior representation is the most significant gap in representing political, military, economic, social, information and infrastructure (PMESII) aspects of the operational environment in urban operations. Since human behavior and societal dynamics are far too complex to be adequately represented by a single analytic model, an approach taken by Seitz (2008) and Ferris (2008) is to combine several different models: three simple analytic models of specific aspects of human beliefs or behavior, along with a stochastic simulation model that can capture some of the richness of the operational environment and the mutual interactions among diverse sets of agents. They show that transferring simple analytic models into advanced simulation software developed for other purposes can bring unpredictable difficulties.

In this section, we briefly describe some of Seitz's findings regarding the incorporation of social network and attitudinal influence models into an agent-based simulation. Pythagoras (Northrup Grumman, 2008) was chosen because it is an existing government-owned, open-source, simulation modeling platform, which had recently been enhanced by the developer to remove some of its prior limitations in its abilities to map certain sorts of human behavior.

The broad topic of representing urban cultural geography is an ongoing effort for the U.S. Army Training \& Doctrine Command Analysis Center-Monterey (TRACMTRY). Seitz's work is but one of several approaches (including the development of new simulation modeling platform) that TRAC is investigating, and many other agencies and researchers are seeking ways to gain insights from other models of stability and support operations.

\subsection{Social And Attitudinal Influence}

\subsubsection{Social Network Structure}

A society consists of a great number of social classes. Members of these classes share the same ideas, thoughts and beliefs, and are connected within a social network. The reasons for belonging to a social network can be very different. They can depend on birth, education, profession, religion, hobbies, politics, and more. Social network the- 
ory tells us that people influence, and are influenced by, others within the same network (see, e.g., Friedkin 1990; Krackhardt 1990).

Social networks are never homogeneous, but contain different types of members with different connections to external networks. This allows fluid information exchange among interconnected networks, so influences on one network can eventually diffuse into the society at large. Membership in a specific social network can vary over time, as individuals' ties to a network weaken and eventually break, or new individuals are drawn in.

Color is the feature in Pythagoras that expresses the agent's affiliation. The attitude toward the host nation (HN) is expressed as the agent's "blueness" on a scale of 0 to 255 . A high blue value indicates a positive attitude toward the $\mathrm{HN}$, and a low value of blue indicates a negative attitude.

In Figure 4, we attempts to portray a minimal set of social networks that might be needed to model a civilian population for stability, support, and reconstruction operations. We are not attempting to portray a realistic representation of a particular region or country, but instead to show how attitudes might be used to identify the social networks for a particular individual within a society battling insurgency. We define several networks in the model; some networks are disjoint, but others overlap. The insurgency network ranges from 0 to 25 , the network of civilians partial to insurgency from 25 to 127, the network of civilians partial to $\mathrm{HN}$ from 127 to 230, and the soldiering network from 230 to 255 . A neutral network connects civilians with mildly different attitudes toward the HN. Two family networks-one for those initially leaning towards the $\mathrm{HN}$, the other for supporters of the insurgency-span the entire spectrum.

\subsubsection{Influence Within Networks}

In Pythagoras, communication devices are equipped with attribute changers that allow communicating agents to influence one another. An agent can have many such devices, so we use a different device for each networks. Still, all social networks are not equally effective. An agent may be strongly influenced by those in one network, and (at the same time) weakly influenced by those in another network. We implement this in Pythagoras by changing the effectiveness of the communications devices. For example, an agent with an initial attitudinal stance represented by 178 in blueness lives in the color-bin between 168 and 200 and therefore takes part in two networks: his family network and the network of civilians partial to HN. Because 178 is the initial blue value for this subpopulation, the effectiveness for the participation in the family network is set to the maximum possible value of $90 \%$. Our modeling assumption is that even in a family, the communication is not perfect. If this agent's blueness drops to 130, then the effectiveness of the family communication channel will drop to $50 \%$.
In all, there are 14 color-bins implemented, representing the different combinations of social networks an agent can participate in and the effectiveness of the communications within these social networks. These combinations can be seen in Figure 4 on an imaginary vertical axis. For example, an agent with blueness 110 possesses the "Network of civilians partial to insurgency, blueness between 25 and 127" (pink) and his "Subpopulation family network" (orange). The values separating these color-bins are called "triggers" since when these values are reached, they trigger an agent to adopt different behaviors or change one or more communications devices. Most communication devices are two-way devices, allowing every member of a network to talk with (and influence) every other member. Family networks have a slightly different setup. To map the stronger influence a family leader might have on his family members in a patriarchal society, we provide each family leader with a one-way device for talking only; the leader can influence his family, but will not receive direct feedback.

In addition to colors, Pythagoras has ten "attributes" that can represent a person's core beliefs, and attribute changers can now be used with weapons, terrain, or communications devices. We define four attributes for attitudinal effects in this study, representing the core beliefs of religion, infrastructure, security, and economic security. With the built-in attribute changer device, the values of these beliefs can be changed, and because a change in a belief will alter a person's behavior, this should consequently change the value of an agent's blueness. There is no means in Pythagoras 2.0.0 to automatically change the blue value when an attribute value changes, so we tie these two together-in our model, a sufficient amount of change in the attribute values is responsible for a change in an agent's color status. Not all core beliefs have the same importance for a person, so we also introduce the concept of weighted attributes. This concept takes care of these differences and changes the attitude of an agent depending on his weighting of his core beliefs. All communications that actively transfer influence through the network possesses an attribute changer.

If a member of this subpopulation gradually changes his attitude towards the HN over time and becomes a supporter of the insurgency, he will proceed through different color-bins, and in each bin the agent possesses different communication devices with different settings. Losing and gaining network participations is possible. He adds the neutral network component but does not give up his connection to those partial to the HN when his blueness is between 127 and 138 . He keeps this neutral network component, stops talking to civilians partial to the $\mathrm{HN}$, and starts talking to civilians partial to the insurgency when his blueness falls between 116 and 127. Later on his way to becoming a terrorist he will lose this neutral connection as well as obtain access to the insurgency network; although he still possesses his family network, its effectiveness will drop dramatically. 


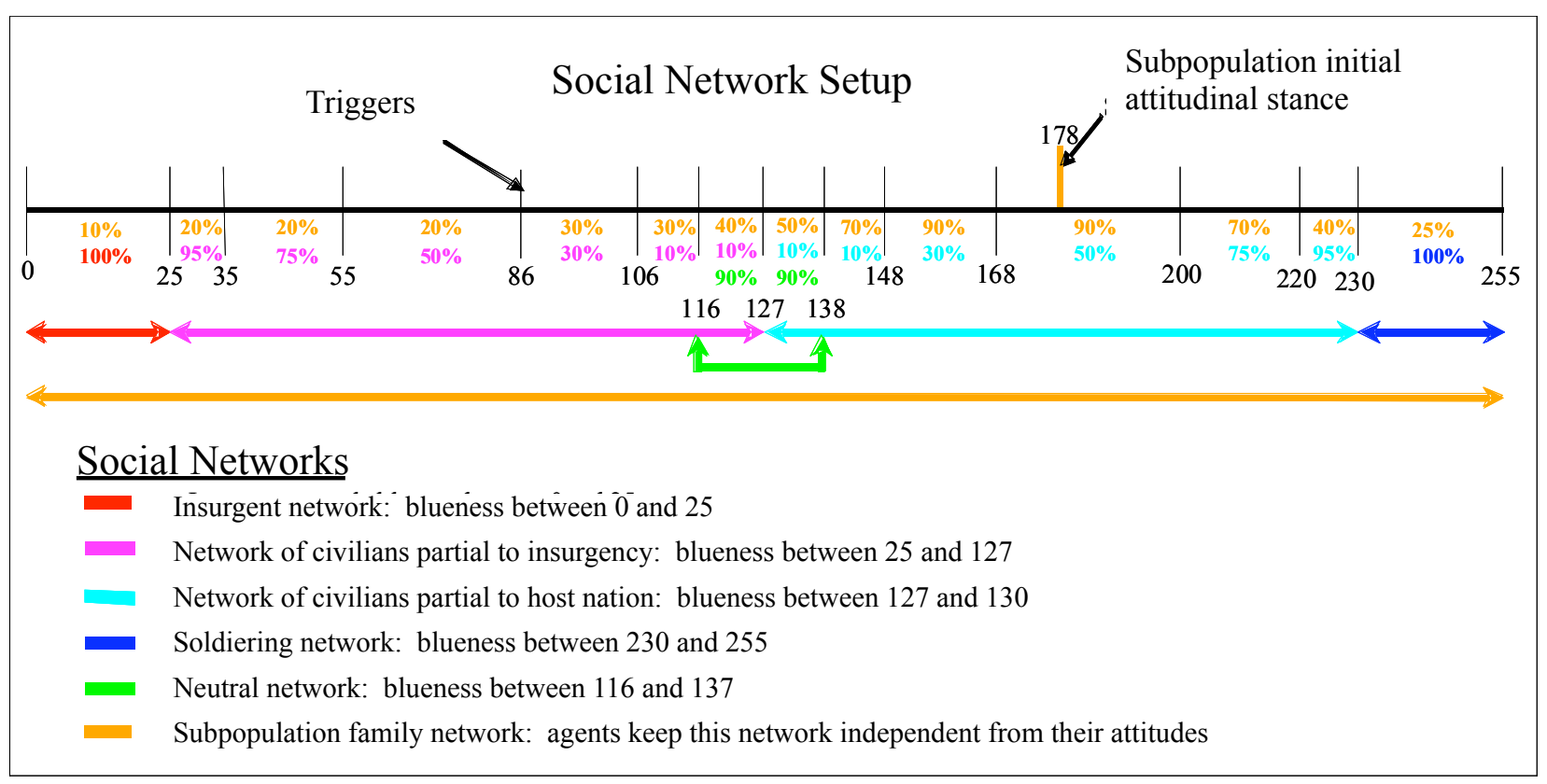

Figure 4: Social network setup. The colored bands indicate different social networks, and the percentages indicate the effectiveness of communications for the corresponding networks.

\subsection{Subpopulations}

Even after the networks are defined, not all members of a subpopulation have the same thoughts and beliefs. Not all attendees of a specific mosque are soldiers, plumbers, or terrorists. But certain percentages of them are similar, and the subpopulation's distribution reflects this. In the Pythagoras model, we start by considering two subpopulations, $S_{1}$ and $S_{2}$, that could represent two different ethnic, religious, or political groups. Suppose $S_{1}$ initially leans toward the $\mathrm{HN}$, while $S_{2}$ initially leans toward the insurgency. (All agents within $S_{1}$ will share the same "family" network, and similarly for $S_{2}$, in our implementation.) These two subpopulations can be further divided into smaller parts, depending on whether the agents are taking part in insurrection, production, or soldiering activities to support their families. This is important if economic activities are going to be incorporated into the model.

For example, $S_{1}$ might begin with $5 \%$ of its agents leaning toward the insurgency but in the production force, $90 \%$ of its agents in the production force initially leaning toward the $\mathrm{HN}$, and $5 \%$ of all agents employed as soldiers. In contrast, $S_{2}$ might begin with $15 \%$ of its members insurgents, $55 \%$ of its members in the production force favoring the insurgency, $28 \%$ in the production force favoring the $\mathrm{HN}$, and $2 \%$ employed as soldiers. In an analytic model, such as the diffusion models used for social networks, these smaller subpopulations are assumed to be homogeneous. In a simulation model, these restrictions are not necessary.
For example, distributions $F_{1}$ and $F_{2}$ could be used to set the initial blueness values for agents in subpopulation $S_{1}$ and $S_{2}$, respectively.

We implement one special agent in each subpopulation called the Leader, who serves the function of a clan leader in a tribal (patriarchal) society. Each leader possesses a one-way communications channel, along which he passes influence to his subpopulation without being influenced in return. We also provide the leaders with more powerful attribute changers than their followers, to represent the greater influence that leaders have over their subpopulations.

\subsection{Experiments And Results}

In the model development phase and initial tests, some unexpected results occurred. These led to Seitz (2008) and Ferris (2008) simplifying the model to track down reasons for this behavior. One simplification was to reduce the number of agents to two: one leader and one follower. A nearly-orthogonal Latin hypercube (NOLH, see Cioppa and Lucas 2007) was used to vary the attribute change values (in a single time step) for each of the four attributes over the range 1 to 20 per time step (between $0.1 \%$ and $2.0 \%$ of the total value for the attribute).

Figure 5 shows what should happen to the leader's and follower's attitudes over time, after a series of strong, positive influences on the leader. Yet as Figure 6 shows, the combination of Pythagoras' time-step behavior and limitations imposed on trigger events meant the actual attitude 
values were radically different - the average attribute value in the simulation converged to just under 300 (an error rate over $70 \%$ ), rather than reaching the desired value of 1000 , because of stable oscillatory patterns that emerge as a function of the time step, attitude change values, and trigger events.

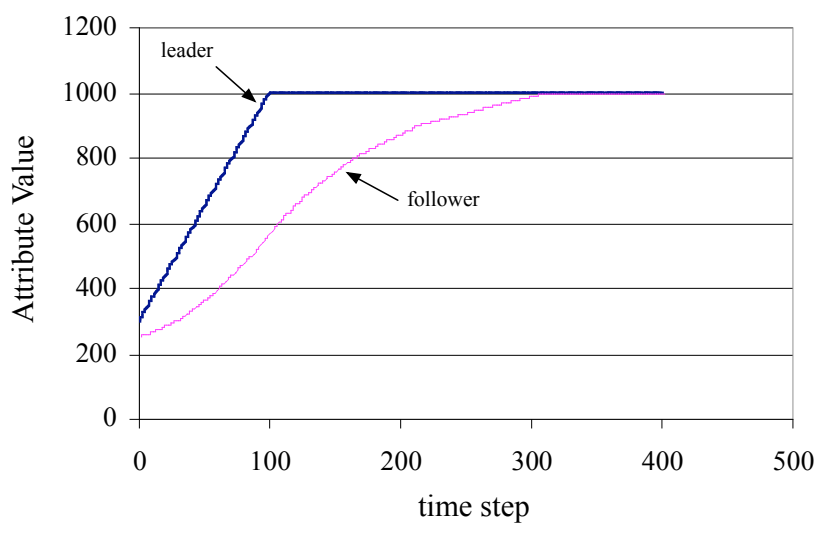

Figure 5: Calculated correct attribute values for a leader and a follower after a series of strong, positive influences on the leader.

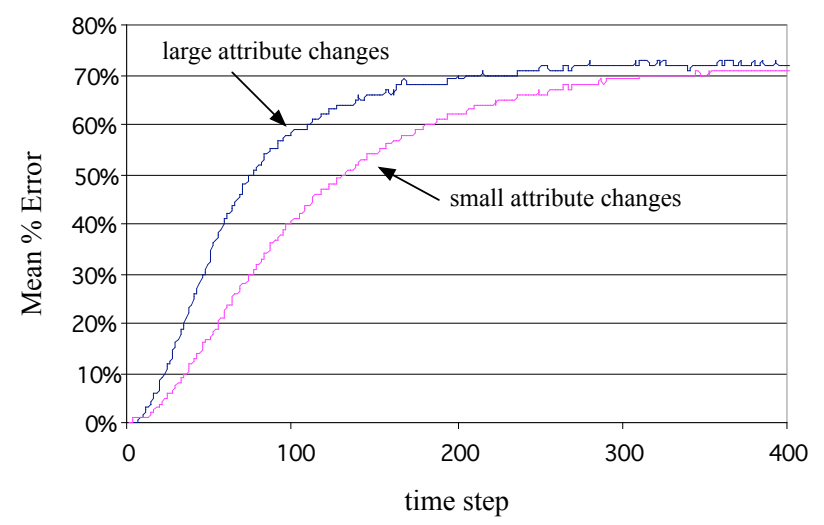

Figure 6: Mean \% error in attribute values over time, for small and large attribute changes per time step.

\subsection{Summary}

Because Pythagoras is a combat model, it can easily represent all parts of a stabilization operation that are related to any kind of military actions. Patrolling areas, hunting down terrorists, responding to terrorist attacks, and so forth are easily to model; this part of the attitudinal model is wellmodeled. Even global actions that influence a population like mass media or taxes can be modeled and analyzed, whether the entire populace is under this influence at the same time or only parts of it in different locations are affected. So influences from the outside that act on single agents, groups of agents, or all agents at once can be mapped.

There are other aspects of a stabilization operation and human behavior that are not easy to map to Pythagoras, including the dispersion of influence through social networks we describe in this paper. The detailed results and findings in Seitz (2008) show a way to enhance the capabilities of Pythagoras 2.0.0, so the software could be used by the U.S. Army and Marine Corps for more sophisticated analyses of stabilization operations. But they also demonstrate that it might be better to use more than one simulation software platformalong with more than one version of any component analytic modelsto represent and predict human behavior.

This study clearly shows that experimental design is a valuable tool during model development. It allows the analyst to explore a wide variety of situations and identify those that need to be investigated in greater detail. In the end, this will help the decision maker to come up with better decisions regarding stabilization operations and other issues critical to global security.

\section{INFORMATION NETWORKS SUPPORTING LOGISTIC SUSTAINMENT OPERATIONS}

The previous two examples considered the implementation of networks in abstract models of a combat operation and civilian populations. Yet another aspect of the Department of Defense's transformation to network-enabled forces is the role that networks play at the tactical logistics level.

Network-enabled and information systems provide the visibility of node and mode status in a shared Logistics Common Operating Picture (LCOP). Currently, there are a variety of communications systems employed that enable distribution operations; however, in some instances at the Soldier level these capabilities do not exist. Accordingly, individual units and commands have supplemented their units with a myriad of commercial, off-the-shelf products as system and network enablers to fill current networkenabled capability needs.

Recognizing the need for supporting these operations, TRAC-MTRY is conducting a study to identify networkenabled capability gaps for Combat Service Support (CSS) Soldiers, as well as potential solutions to fill those gaps.

In this section, we briefly summarize the work of Baez (2008), who explores the use of the Logistics Battle Command (LBC) model to assess the effects of Soldier-level, network-enabled capabilities on cargo operations at a truck terminal within a sustainment base. The LBC model, developed by TRAC-Monterey, is a low-resolution, objectoriented, stochastic, discrete-event model that enables the analysis of sustainment battle command scenarios. The intent is to determine the types of operational insights that LBC can provide - specifically, in terms of quantifying the 
impacts that network-enabled capabilities on the logistics sustainment operations.

\subsection{Model of Logistics Sustainment}

The scenario modeled is one of Army Transportation Soldiers performing cargo terminal operations at a Centralized Receiving and Shipping Point (CRSP) within a Forward Operating Base (FOB). These operations support regular sustainment convoys, that deliver equipment and supplies to their final destinations. CRSPs (in current operations) are different in size with different layouts. Nevertheless, most CRSPs are composed of an operations center, a palletized cargo area, a customer container area/lanes, unit vehicles or rolling stock area/lanes, an area for battle-damaged vehicles, and an empty container collection point (EECP) area to ensure fluid vehicle movement within the CRSP yard. Figure 7 (from Center for Army Lessons Learned 2007) shows a typical CRSP layout.

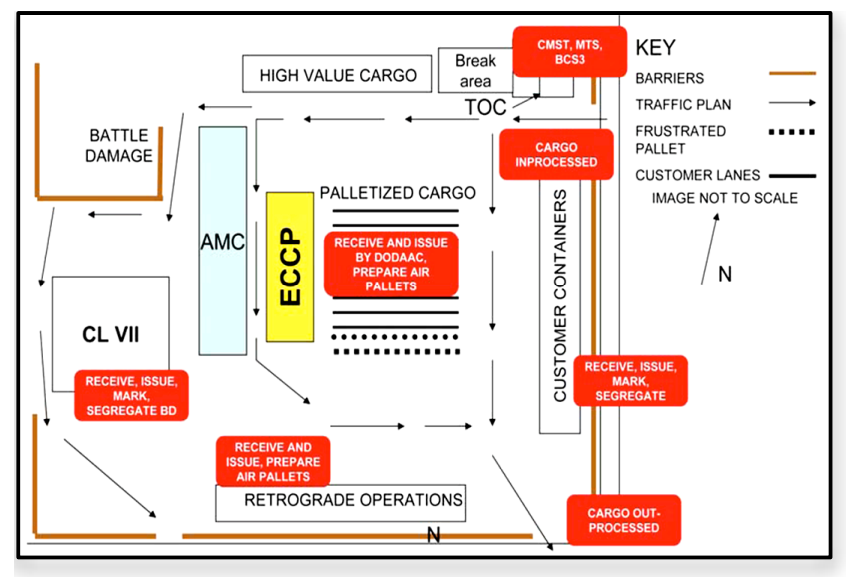

Figure 7: CRSP layout.

Typically, the customer container area contains an inbound and an outbound lane. Containers that have completed the final leg of their movement or are to be picked up at the CRSP are staged in the inbound container lane. Containers that will continue their onward movement are staged in the outbound container lane. Rolling stock is usually treated in the same manner as containers, with the exception that the rolling stock lanes require a much larger area. A palletized cargo area allows for pallets to be built and convoys to come in and stage so that one side loads and the other side offloads. The battle-damaged vehicle area segregates these vehicles from other retrograde cargo. ECCPs are established for cross loading containers used for retrograde cargo arriving from the FOBs, and to exchange any carrier-owned and leased containers with government-owned containers. Finally, the operations center is the central location where all of the cargo entering and exiting the yard is processed and ac- counted for; additionally, the operations center synchronizes the CRSP efforts to ensure uninterrupted operations.

Three different network topologies are of interest (Figure 8). The Hierarchical topology represents the existing physical laydown and connectivity for the current force. Specifically, operations in the CRSP are largely governed by paper-based manifests, radio reports, and radio frequency (RF) technology capabilities. The CRSP operations center can access the LCOP and develop detailed plans based on the ITV data, but those plans are made available to the container, pallet, and rolling stock lane in an ad hoc manner by radio, face-to-face, and paper message processes. These ad hoc manner methods are time consuming and operationally non-responsive. In the Star topology, each of the four nodes of the network within the CRSP is connected to the network-centric LCOP node with a point-to-point link. The resulting structure has four communications channels in a hub-and-spoke arrangement. The Hierarchical-Star topology, as its name implies, combines the first two topologies together to form a more complex network.

\subsection{Measures of Effectiveness}

Baez examines three measures of effectiveness: velocity, reliability, and visibility. Velocity is expressed as the mean time in CRSP, which includes waiting time plus time receiving service. As the mean time in CRSP decreases, velocity increases. Reliability is the degree of assurance or dependability that CRSP operations will consistently meet cargo demands under established conditions to specified standards. Reliability measures the variability of the mean time in CRSP and the mean difference in area of visibility. Visibility represents the capacity to determine the status, location, and direction of flow of materiel. Visibility requires the availability of timely, accurate, and usable information essential to the maintenance of the LCOP with the overall joint distribution stakeholders. It quantifies the mean difference in the area between the ground-truth stock levels at the CRSP lanes, and the levels reported at the LCOP.

Since queueing theory certifies that resource utilization and flow rates (convoys per hour) determine delays in the system, our analysis focuses on these factors. After the experiment was complete, these two factors were combined into a single term called traffic intensity. This does not directly correspond to the traffic intensity in a mathematical model of, say, an M/M/1 queueing system, where a traffic intensity of 1.0 or more leads to infinite queue build-ups. Nonetheless, higher traffic intensities are associated with more congestion in the CRSP. (An alternative experimental design could vary convoys per hour and traffic intensity, rather than conveys per hour and resource utilization, as the factors to facilitate the analysis; see Kleijnen et al., 2005). 


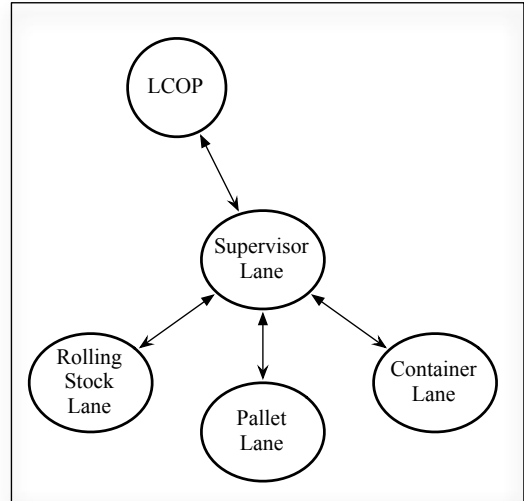

Hierarchical network

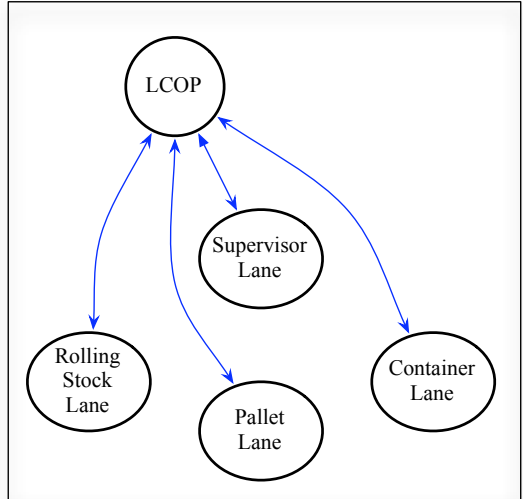

Star network

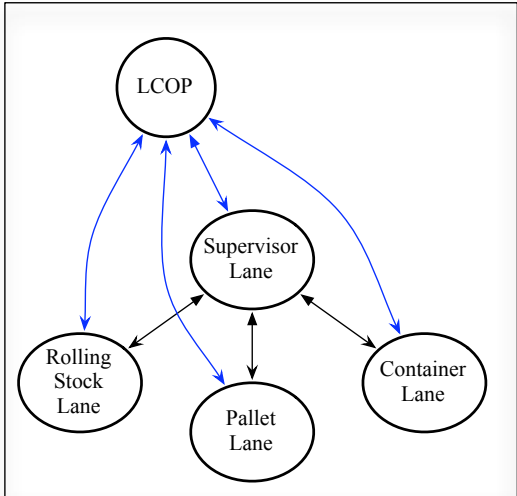

Hierarchical-star network

Figure 8: Three different network topologies explored in the logistics sustainment simulation.

\subsection{Experimental Design}

The factors are derived directly from concept-specific attributes listed in the Net-Centric Operational Environment Joint Integrating Concept (JS, 2005) and input from subjectmatter experts. The decision factors considered are the availability and accuracy of in-transit visibility information (ITV-available and ITV-accuracy), the rate at which a provider or CRSP lane updates the LCOP (LCOP update), probability of communications, latency, and communication relay capability. These factors all potentially influence network capability for the scenario, and represent choices that could be implemented in the CRSP. The noise factors are resources available, convoys per hour, and convoy composition. Varying these factors allows for examining the impact of network capability aspects across a broader range of potential operating conditions.

In all, ten replications of each of 771 different scenarios (design points) were used to generate results -257 for each of the three dissimilar network structures. The system starts in an empty-and-idle state, generates convoy arrivals at the appropriate rate up to a maximum number, and simulates until all these convoys have been processed.

\subsection{Results}

We present a few results to indicate the types of information that can be obtained from such a study (see Baez 2008 for more detail). First, contour plots of the Velocity (mean time in CRSP) as functions of the traffic intensity and in-transit visibility are shown in Figure 9 (best viewed in color). The red areas in the lower right-hand corners correspond to very poor velocity (high mean time in CRSP). The Hierarchical network (on the left) performs worst, and the Hierarchical-Star network (on the right) performs best. All three network topologies have some scenarios where the CRSP is unable to handle the volume of work and the mean time in CRSP is excessively high.

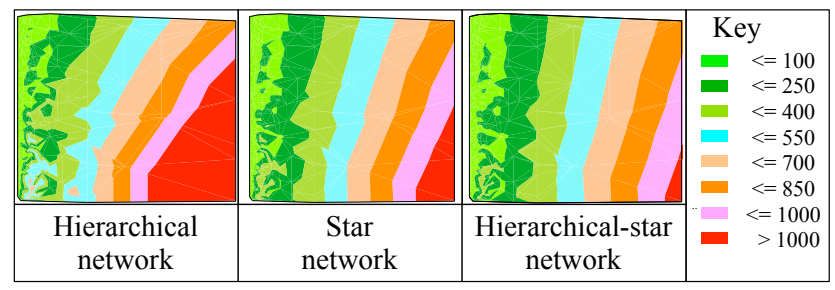

Figure 9: Contour plots of the mean time in CRSP as functions of traffic intensity ( $x$-axis) and in-transit visibility data availability ( $y$-axis).

A closer look provides additional insight into this behavior. Naturally, the system contains an initial transient period. The CRSP starts in an empty-and-idle state, so early convoys will experience fewer congestion-related delays than later convoys. Because LBC operates on a first-come, first-served basis, a particular cargo-processing time is not influenced by any cargo that arrives later. If the initial transient period is short, then the steady-state processing time distributions may be reached before the simulation terminates.

In Figure 10, we show traces of the time in CRSP for each convoy for three replications of three different design points. The left-most plot shows that Design Point 1 appears to achieve steady-state with no warm-up period, although there is a large amount of variability in the system. The center plot shows that Design Point 31 has a longer warm-up period and much greater variability, as seen by the differences between the traces for the three replications; it also reveals strong, positive correlation of the mean times in CRSP across convoys. The right-most plot is on a different scale, but shows curious behavior: the mean times in CRSP rise steadily, then drop slightly, 
and then rise again. When the mean time in CRSP keeps increasing for the later convoys, this indicates that the CRSP is incapable of handling the level of traffic with its current resources.

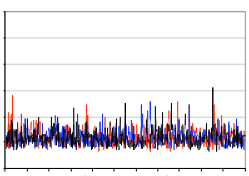

'Desigñ Pöiñt'-'

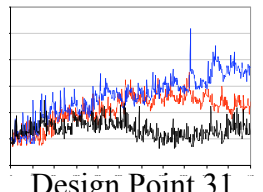

Desigñ Point 31

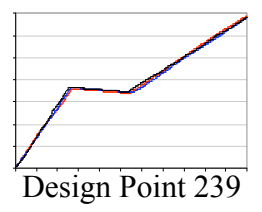

Figure 10: Trace results of time in system for the first three replications of three different design points.

The dip in the right-most plot in Figure 10, as well as some other unusual behavior, needs to be examined further before relying too heavily on operational insights from these results. Nonetheless, Baez's results that the network structure does affect all three measures of effectiveness in the LBC model. A summary of some findings from regression metamodeling and regression tree analyses follows.

Velocity. The most significant factors affecting velocity for all topologies are the traffic intensity and the availability of in-transit visibility (ITV) data. Changing from a Hierarchical to a Star or Hierarchical-Star network improves velocity by $32 \%$ and $42 \%$, respectively, in our model.

Reliability. Reliability is also affected by traffic intensity and ITV data availability. Overall, the Hierarchical structure was the least reliable.

Visibility. The most significant factors influencing visibility differ by the network topology. For the Hierarchical structure, these are the communication relay capability at the supervisor lane, and the probability of communications between the supervisor and the LCOP. For the Star structure, these are the probability of communications between the LCOP and the pallet lane, as well as the LCOP and container lane, and the communications relay capability at the pallet lane. For the Hierarchical-Star structure, these are the communications relay capability at the supervisor lane, the probability of communications between the LCOP and the container lane, as well as the LCOP and pallet lane. Overall, changing from a Hierarchical structure to a Star or Hierarchical-Star structure improves visibility by $43 \%$ and $59 \%$, respectively, in our model.

Typically, architectural analysis based on subjectmatter-expert input is the basis of the capability-based assessment (CBA) process, and modeling and simulation is rarely used. However, the results from this research suggest that modeling and simulation-combined with an efficient design of experiments approach-will result in a more robust process, and add credibility to the CBA findings.

\section{CONCLUDING REMARKS}

The three studies summarized in this paper consider different aspects of information networks at different levels of abstraction. But in all cases, these studies do not stop after obtaining results for a single, baseline model. Instead, they use experimental designs to conduct broad explorations of the simulation models' behaviors under various conditions. This use of experimental design is very powerful. It provides much broader insights into the model behavior, which can assist in verifying that the model is coded properly, and providing assurance that the model is suitable for its intended use. It allows many factors to be studied simultaneously, so interactions among factors that might be missed with a trial-and-error approach can be identified and exploited.

Modeling intangibles like the value of information, human attitudes and social dynamics, and the impact of new technologies and communication structures is a difficult task-but one that cannot be ignored. Decision-makers faced are faced with difficult questions about the best equipment, tactics, techniques, and procedures to use in current operations, and they must also look to the future to determine how to build, organize, equip, maintain, and, if necessary, employ their military and security forces. These dynamic, rapidly-changing environment adds complexity. For instance, we may want urban cultural geography models capable of being quickly modified to support a range of stability and support operations-from sectarian conflict within a region, to stability and support operations following a natural disaster. What is clear is that with the increasing reliance on new technologies and networkenabled operations, incorporating information networks into the simulation models used to support these decisions is extremely important.

\section{ACKNOWLEDGMENTS}

This work was partially supported by TRAC-MTRY.

\section{REFERENCES}

Baez, F. R. 2008. Combat service support soldier network enabled operations (CSNEO). M.S. thesis, Operations Research Department, Naval Postgraduate School, Monterey, California.

Bracken, J. and R. E. Darilek. 1998. Information superiority and game theory: The value of information in four games. PHALANX 31(4): 6-7, 33-34.

Center for Army Lessons Learned. 2007. Centralized receiving and shipping point handbook. CAA Publication No. 07-33. Fort Leavenworth, Kansas: Center for Army Lessons Learned. 
Cioppa, T. M., and T. W. Lucas. 2007. Efficient nearly orthogonal and space-filling Latin hypercubes. Technometrics 49(1): 45-55.

Ferris, T. P. 2008. Modeling methodologies for representing urban cultural geographies in stability operations. M.S. thesis, Operations Research Department, Naval Postgraduate School, Monterey, California.

Friedkin, N. E. 1990. Social networks in structural equation models. Social Psychology Quarterly 53: 316-328.

Jackson, J. B. 2008. Exploring the importance of information superiority to the decision maker. M.S. thesis, Operations Research Department, Naval Postgraduate School, Monterey, California.

Joint Chiefs of Staff. 2006. Joint publication 3-13: Information operations. Washington, D.C.: Department of Defense. Available via <wWW. fas. org/irp/doddir/dod/jp3_13.pdf> [accessed June 30, 2008].

Joint Staff. 2005. Net-centric operational environment joint integrating concept. Version 1.0. Washington, D.C.: Department of Defense. Available via www.dtic.mil/futurejointwarfare/conce pts/netcentric-jic.pdf

Kleijnen, J. P. C., S. M. Sanchez, T. W. Lucas, and T. M. Cioppa. 2005. A user's guide to the brave new world of designing simulation experiments. INFORMS Journal on Computing 17(3): 263-289.

Krackhardt, D. 1990. Viscosity models and the diffusion of controversial innovations. In Dynamics of organizations: Computational modeling and organizational theory, ed. A. Lomi and E. R. Larsen, 243-268. Cambridge, Massachusetts: MIT Press.

Northrup Grumman. 2008. Pythagoras user's manual. Version 2.0. Arlington, Virginia: Northrup Grumman.

Seitz, T. 2008. Representing urban cultural geography in stabilization operations: Analysis of a social network representation in Pythagoras. M.S. thesis, Operations Research Department, Naval Postgraduate School, Monterey, California.

Washburn, A. R. 2001. Bits, bangs, or bucks? The coming information crisis. PHALANX 34(3): 6-7, 24-27 (Part I) and 34(4): 10-11, 31-32 (Part II).

Washburn, A. R. 2003. Two-person zero-sum games. 3rd ed. Hanover, Maryland: INFORMS.

\section{AUTHOR BIOGRAPHIES}

DARRYL K. AHNER is an Assistant Professor at the United States Military Academy. A Lieutenant Colonel in the United States Army, he received his Ph.D. in Systems Engineering (Operations Research) from Boston University. His e-mail address is <darryl. ahnereusma. edu>.
JONATHON K. ALT is a Major in the U.S. Army. After finishing his current tour in Iraq, he will return to his position as an Operations Research Analyst in the U.S. Army Training and Doctrine Command Analysis Center in Monterey, California (TRAC-MTRY). He has an M.S. in Operations Research from the Naval Postgraduate School, and received the Edward Kelleher Director's Award for Research Excellence from TRAC-MTRY for his thesis work. His e-mail address is <jkaltenps . edu>.

FRANCISCO R. BAEZ is a Major in the U.S. Army. He currently serves as an Operations Research Analyst in the U.S. Army Training and Doctrine Command Analysis Center in Monterey, California. He received his B.S. in Logistics from the University of Puerto Rico, and his M.S. in Operations Research from the U.S. Naval Postgraduate School. His email is <frbaezto@nps. edu>.

JOHN JACKSON is a Marine Corps Intelligence Officer. A Major in the Marine Corps, he has a Masters Degree in Operations Research from the Naval Postgraduate School. He has served in a number of operational billets in Bosnia, Iraq and the western pacific. He is currently serving as an operations analyst for Joint Forces Command J9 Future Concept Development and Experimentation in Suffolk, VA. His e-mail address is <jacksonj3@hotmail. com>.

SUSAN M. SANCHEZ is a Professor in Operations Research at the Naval Postgraduate School, and Co-Director of the Simulation Experiments \& Efficient Design (SEED) Center for Data Farming. She also holds a joint appointment in the Graduate School of Business \& Public Policy. She has a B.S. in Industrial \& Operations Engineering from the University of Michigan, and a Ph.D. in Operations Research from Cornell. She has been active in various service capacities within the simulation community over many years, and is currently on the WSC Board of Directors. Her web page is <faculty.nps.edu/smsanche/> and her e-mail is <ssanchez@nps. edu>.

THORSTEN SEITZ was born in 1964 in Einbeck, Lower Saxony, where he spent his childhood and his school time. In 1984 he joined the German Navy and served in the Fast Patrol Boat Flotilla as an Operations Officer; in the Destroyer Squadron as Head of Department Logistics and Supply Officer; and in the German Naval Office as Head of Section (HOS) Spare Part Procurement and Coordination/Management of Spare Part Supply, as well as HOS Logistic Information Center. After completing his M.S. in Operations Research at the Naval Postgraduate School he will return to his home country and be the the Commanding Officer of the Material Depot in Wilhelmshaven. His e-mail address is seitzthorstendaol.com>. 